\title{
Condiciones higiénico-sanitarias basadas en las cinco claves de la OMS de los servicios de alimentación de hospitales del departamento de Caaguazú, Paraguay
}

\author{
*Ninfa Fernández ${ }^{1}$, Stella Cabral de Bejarano ${ }^{1}$, Gladys Estigarribiaa, ${ }^{2,3}$, Analía Ortiz², Patricia Ríos ${ }^{2,3}$ \\ ${ }^{1}$ Asociación de funcionarios de la Universidad Nacional de Caaguazú, AFUNCA. Paraguay \\ ${ }^{2}$ Universidad Nacional de Caaguazú, UNCA. Paraguay \\ ${ }^{3}$ Instituto Regional de Investigación en Salud, IRIS. Paraguay
}

\author{
Cómo referenciar este artículo/ \\ How to reference this article:
}

Fernández N, Cabral de Bejarano S, Estigarribia G, Ortiz A, Ríos P. Aplicación de las 5 claves de la OMS para medir las condiciones higiénico-sanitarias de los servicios de alimentación de hospitales del departamento de Caaguazú. Mem. Inst. Investig. Cienc. Salud. 2018; 16(2): 21-31.

\section{R E S U M E N}

La prevención de enfermedades transmitidas por alimentos (ETA) en hospitales, se vincula a la higiene de los alimentos y buenas prácticas de manufactura (BPM). Estudio observacional, descriptivo de corte transversal cuyo objetivo fue evaluar las condiciones higiénico-sanitarias de los servicios de alimentación de los hospitales del departamento de Caaguazú, Paraguay. Se incluyeron todos los hospitales $(n=12)$ con servicios de alimentación con sus correspondientes manipuladores de alimentos $(n=26)$. Se utilizaron cuestionarios elaborados en base a la Resolución 80/96 del Mercosur sobre BPM, las recomendaciones de las "Cinco Claves de la OMS" para la inocuidad de los alimentos, además de los datos socio-demográficos de los manipuladores. El 33,3\% de los responsables de los servicios de alimentación reportó no seguir las recomendaciones sobre lavado de manos, se observó que el $41 \%$ de los basureros de la cocina estaban sucios (clave 1), 25\% de los manipuladores utilizaban los mismos utensilios para alimentos crudos y cocinados, y $41,7 \%$ no aplicaba métodos apropiados en la degustación (clave 2). Todos reportaron cocción total de los alimentos, pero solo el $16,7 \%$ tenía registro de la temperatura interna de ellos (clave 3). Para alimentos con aliños, 83,3\% no limita la exposición a temperatura peligrosa (clave 4). El 33\% no utiliza el sistema FIFO para rotación de alimentos, y $25 \%$ de manipuladores reportó lavar frutas y verduras según indicaciones de inocuidad (clave 5). Se encontraron deficiencias higiénico-sanitarias en los servicios de alimentación probablemente a la escasa formación en BPM de los manipuladores y deficiencias en las regulaciones en los servicios.

Palabras clave: hospitales, servicios de alimentación, condiciones higiénico sanitarias.

\section{Sanitary hygienic conditions based on the WHO five keys of hospitals food services of the department of Caaguazu, Paraguay}

\section{A B S T R A C T}

Prevention of foodborne diseases (FBD) in hospitals is related to food hygiene and good manufacturing practices (GMP). The objective of this observational descriptive crosssectional study was to evaluate the sanitary hygienic conditions of the food services of hospitals of the department of Caaguazú, Paraguay. All hospitals $(n=12)$ with food services and their food handlers $(n=26)$ were included. Questionnaires elaborated based on the Mercosur Resolution 80/96 on good manufacturing practices (GMP), WHO "Five Keys for the safety of food" in addition to socio-demographic data of the food handlers were used. The $33.3 \%$ of those responsible for the food services reported not following the recommendations on hand washing, $41 \%$ of the garbage dumps in the kitchen were dirty (key 1 ), 25\% of the handlers used the same utensils to handle raw and cooked foods and $41.7 \%$ did not apply appropriate methods for tasting (key 2). All reported total cooking of 
the food, only $16.7 \%$ had records of the food internal temperature (key 3 ). For foods with dressings, $83.3 \%$ does not limit the exposure to dangerous temperature (key 4 ). The FIFO is not used in $33.3 \%$ of cases and $25 \%$ of handlers reported washing fruits and vegetables according to safety indications (key 5). Hygienic-sanitary deficiencies in the food services were found, probably attributed to the poor training in GPM of handlers, and poor regulations in the services.

Keywords: hospitals, food services, sanitary hygienic conditions.

\section{INTRODUCCIÓN}

La salud y la vida de las personas dependen en gran parte de la calidad nutricional de los alimentos que consumen diariamente, que a su vez depende de la inocuidad higiénicosanitaria a que estos son sometidos en toda la cadena productiva, desde el campo a la mesa del consumidor ${ }^{(1)}$. Los servicios de alimentación y nutrición de los hospitales tienen como misión dar respuesta a la necesidad de alimentación de los usuarios con alimentos en óptimas condiciones higiénicas y organolépticas en los tiempos establecidos ${ }^{(2)}$.

En los últimos años se ha observado una creciente incidencia de enfermedades transmitidas por los alimentos (ETA) a nivel mundial, ocasionando preocupación en el ámbito de la Salud Pública en cuanto a la eficacia de los sistemas que garanticen calidad e inocuidad de los alimentos que se consume ${ }^{(3)}$. Las ETA son definidas como el "síndrome originado por la ingestión de alimentos y/o agua, que contengan agentes etiológicos en cantidades tales que afecten la salud del consumidor a nivel individual o grupos de población" ${ }^{\prime(4)}$.

La Organización Mundial de la Salud (OMS) informa que cada año unas 2 millones de personas mueren debido a alguna de las más de 200 enfermedades que pueden transmitir los alimentos, desde diarrea hasta cáncer, representando el $25 \%$ de todas las patologías existentes; lo importante es que todas ellas se pueden prevenir ${ }^{(5)}$.

Tras casi un año de consultas con expertos en inocuidad de los alimentos y en comunicación de riesgo, la OMS presentó en el 2001 las cinco claves para la inocuidad de los alimentos, una herramienta para mantener los alimentos seguros, cuyos mensajes básicos son: 1. Mantener la limpieza, 2. Separar alimentos crudos y cocinados, 3. Cocinar completamente, 4. Mantener los alimentos a temperaturas seguras y 5 . Usar agua y materias primas seguras ${ }^{(6)}$.

El Paraguay a través del Decreto 17.056/97 ha internalizado la Resolución 80/96 del Mercosur sobre las "Condiciones higiénico sanitarias y de Buenas prácticas de fabricación para establecimientos elaboradores de alimentos", donde las buenas prácticas de elaboración son los procedimientos necesarios para lograr alimentos inocuos y saludables ${ }^{(7)}$. La regulación general de este derecho constituye el objetivo de la Ley Número 836 - Código Sanitario (1980), Libro II de los alimentos. El Instituto Nacional de Alimentación y Nutrición en coordinación con otros organismos del gobierno y las municipalidades (Ley No.3966/10 Orgánica Municipal), es responsable de dar cumplimiento de este precepto.

A pesar de la administración sanitaria establecida, un estudio mediante una inspección de los servicios de alimentación de hospitales mostró que los mismos no poseen guías estatales oficiales ni controles periódicos por las entidades responsables, por lo que estos centros se manejan en forma independiente con reglas propias ${ }^{(9)}$. La falta de cumplimiento de las disposiciones establecidas y la carencia de conocimientos técnicos básicos sobre la inocuidad por parte de quienes preparan alimentos, se puede considerar como uno de los factores que más contribuyen a las ETA ${ }^{(1)}$. Esta situación motivó el desarrollo del presente trabajo y se espera que los resultados sirvan como una línea basal de información sobre las condiciones higiénico-sanitarias de los servicios de alimentación de hospitales públicos y privados del departamento Caaguazú, con el fin de contribuir al análisis del problema, constituirse en insumo para la priorización de acciones públicas en áreas de mayor riesgo y/o realizar estudios complementarios para el aporte de evidencias científicas.

\section{MATERIALES Y MÉTODOS}

Estudio observacional, descriptivo de corte transversal con enfoque cuantitativo en el cual se analizaron las condiciones higiénico-sanitarias de todos los hospitales, públicos, públicos autárquicos y privados con servicios de alimentación para usuarios interno y externo del $V$ departamento Caaguazú y el perfil socio demográfico de 26 manipuladores de alimentos de estos hospitales. Los establecimientos de salud seleccionados están ubicados 
en los municipios de Cnel. Oviedo, Caaguazú, Santa Rosa del Mbutuy, Juan Manuel Frutos, J. Eulogio Estigarribia, Repatriación, Yhú y Cecilio Báez, según ordenamiento territorial del departamento.

La recolección de datos sobre las condiciones higiénico-sanitarias, equipamiento, almacenamiento y prácticas cotidianas de funcionarios se realizó a través de un cuestionario estructurado, aplicado a los funcionarios responsables de los servicios de alimentación, y observación de la infraestructura del establecimiento. La recolección de los datos del manipulador de alimentos se realizó a través de una entrevista personal, con aplicación de un cuestionario estructurado para recabar datos socio demográficos que incluían sexo, edad, escolaridad, formación en educación sanitaria, función laboral, ingreso y antigüedad en el servicio entre otros.

Las preguntas de la encuesta fueron adaptadas de los cuestionarios sobre procedimientos operativos estandarizados de la USDA, Servicio de Alimentación y Nutrición \& Instituto Nacional de Administración de servicios de alimentos, $2005^{(9)}$, así como a las disposiciones consignadas en la Resolución 80/96, Reglamento técnico Mercosur sobre las condiciones higiénico sanitarias y de buenas prácticas de elaboración para establecimientos elaboradores/industrializadores de alimentos, y los aspectos agrupados en función a las 5 Claves de la OMS para la inocuidad de los alimentos. Se tuvieron en cuenta las variables relacionadas con el emplazamiento, la infraestructura de las áreas de manipulación de alimentos, abastecimiento de agua, la higiene del personal y requisitos sanitarios. En el área de preparación de alimentos las variables relativas a la recepción, almacenamiento, producción y distribución, equipos e implementos, superficies de trabajo, cumplimiento de procedimientos de limpieza y desinfección, disposición de basuras y control de plagas. Se obtuvo información del proceso productivo mediante la observación directa y prácticas reportadas.

En la valoración del cuestionario solo hay dos puntuaciones, a cada opción correcta se le asignó valor uno y a cada respuesta incorrecta un único valor cero. Los datos compilados en los cuestionarios se cuantificaron en las planillas Microsoft Excel y analizados con Epi Info, Versión 7 (CDC, 2011).

\section{RESULTADOS}

Se presentan los resultados de las observaciones en los aspectos higiénico-sanitarios de los servicios de alimentación de 12 establecimientos de salud, de los cuales el 66,66\% correspondió al sector público, $16,66 \%$ público autárquico y $16,66 \%$ privado. Los 26 manipuladores de alimentos correspondieron a instituciones públicas $(38,46 \%)$, público autárquico $(34,61 \%)$ y privadas $(26,93 \%)$. Las características de las instalaciones de los servicios de alimentación se describen en la Tabla 1.

Tabla 1: Características de las instalaciones de los servicios de alimentación $n=12$

\begin{tabular}{lll}
\hline Características & $\mathbf{n}$ & $\mathbf{\%}$ \\
\hline Ubicación adecuada en zona libre de olores objetables y contaminantes & 11 & 91,66 \\
Abastecimiento de agua potabilizada & 11 & 91,66 \\
Evacuación de efluentes y aguas residuales a red pública & 7 & 58,33 \\
Construido de materiales duraderos, fáciles de mantener y limpiar & 8 & 66,66 \\
Ventanas y puertas que dan al exterior están protegidas & 2 & 16,66 \\
Instalaciones eléctricas empotradas & 3 & 25 \\
Buena iluminación & 3 & 25 \\
Recursos para extracción de humo y/o vapor & 8 & 66,66 \\
Ausencia de animales domésticos & 11 & 91,66 \\
Baño exclusivo para manipuladores & 5 & 41,66 \\
Baño para manipuladores con inodoro, lavatorio y agua corriente & 8 & 66,66 \\
Depósito de basura ordenado y las basuras embolsadas & 10 & 83,33 \\
Cuenta con un programa de control bajo licencia de operador oficial de plagas & 8 & 66,66 \\
\hline
\end{tabular}


Características de higiene personal y requisitos sanitarios. El $50 \%$ de las manipuladoras utilizan uniforme de trabajo completo, el $33,3 \%$ de las manipuladoras reportan lavado correcto de manos, aunque solo el 16,66\% dispone de los insumos necesarios, en ningún establecimiento se cuenta con toallas desechables para el secado de manos. Se constató libro de registro de enfermedad en $50 \%$ de los servicios. Las características de higiene personal y requisitos sanitarios se describen en la Tabla 2.

Tabla 2. Características de higiene personal y requisitos sanitarios. $(n=12)$

\begin{tabular}{lll}
\hline Características & $\mathbf{n}$ & $\mathbf{( \% )}$ \\
\hline Uniforme de trabajo completo & 5 & 50 \\
Uniforme de trabajo limpio & 8 & 66,66 \\
Manos lavadas correctamente & 4 & 33,33 \\
El lavamanos cuenta con los insumos necesarios & 2 & 16,66 \\
Manos con objeto de adorno y joyas & 6 & 50 \\
Lava sus manos después de toser o estornudar & 7 & 58,33 \\
Las manos están protegidas a prueba de agua en caso de heridas y quemaduras & 2 & 16,66 \\
Uñas cortas y limpias & 10 & 83,33 \\
Secado con toallas desechables & 0 & 0 \\
Indicación visible de cómo lavarse las manos & 4 & 33,33 \\
Capacitación en los últimos dos años sobre higiene personal & 4 & 33,33 \\
Capacitación en los últimos dos años sobre higiene de alimentos & 4 & 33,33 \\
Registro de enfermedad de manipuladores & 6 & 50 \\
\hline
\end{tabular}

Aplicación de las 5 Claves de la OMS en la higiene de la elaboración de alimentos. La higiene en la elaboración con aplicación de los principios de las 5 Claves de la OMS se describe en la Tabla 3. Clave 1. Mantener la limpieza. El 66,7\% de los responsables de los servicios de alimentación reportó que las manos son lavadas con la frecuencia recomendada. Respecto al basurero de la cocina, en $41,7 \%$ de los casos se observó que el mismo estaba sucio. Clave 2. Separar alimentos crudos y cocinados. Los alimentos crudos y cocinados se mantenían separados en el $66,7 \%$. El $75 \%$ de los manipuladores utilizaba utensilios distintos para manipular alimentos crudos y listos para comer. El 58,3\% aplicaba métodos apropiados en la degustación. Clave 3. Cocinar completamente. El $100 \%$ de los alimentos se cocinan hasta asegurar que la parte interna llegue a $70{ }^{\circ} \mathrm{C}$, y $16,7 \%$ mantiene registros de la temperatura interna de los alimentos. Clave 4. Mantener los alimentos a temperatura segura. El $16,7 \%$ de los alimentos con aliños se preparaba en pequeñas cantidades para limitar exposición a temperatura peligrosa El $58,3 \%$ de los manipuladores realizaba la descongelación en el refrigerador. Clave 5. Usar agua y materia prima seguros. En $91,7 \%$ de los comedores la procedencia del agua es potable. En la rotación de alimentos, el $66,7 \%$ utilizaba el sistema FIFO. El $25 \%$ de los manipuladores lava las frutas y verduras siguiendo las indicaciones de inocuidad. 
Tabla 3. Aplicación de las 5 Claves de la OMS en la elaboración de alimentos. $n=12$

\begin{tabular}{lll}
\hline Características & n & \%) \\
\hline Clave 1. Mantener la limpieza & & \\
Las manos son lavadas con la frecuencia recomendada & 8 & 66,7 \\
Utensilios de cocina y superficies en contacto con alimentos son lavados y desinfectados & 11 & 97,1 \\
antes de cada uso. & 10 & 83,3 \\
Los alimentos están adecuadamente protegidos & 7 & 58,3 \\
El basurero de la cocina está limpio & 10 & 83,3 \\
El basurero de la cocina tiene bolsa de polietileno & 9 & 75,0 \\
El basurero de la cocina tiene tapa & &
\end{tabular}

\section{Clave 2. Separar alimentos crudos y cocinados}

Los alimentos crudos y cocinados están separados

Se utilizan utensilios distintos para manipular alimentos crudos y listos para comer $\quad 9 \quad 75,0$

Los equipamientos y utensilios (incluyendo tablas de picar) están limpios y desinfectados $10 \quad 83,3$ entre usos

Degustación apropiada de alimentos

\section{Clave 3. Cocinar completamente}

Los alimentos se cocinan hasta asegurarse que se cocinen bien

Clave 4. Mantener los alimentos a temperaturas seguras

La temperatura de los alimentos refrigerados está por debajo de $5^{\circ} \mathrm{C} . \quad 11 \quad 97,1$

$\begin{array}{lll}\text { La descongelación se realiza en el refrigerador } & 7 & 58,3\end{array}$

Alimentos con aliños como mayonesa, salsa de ajo se preparan en pequeñas cantidades $2 \quad 16,7$

para limitar exposición a temperatura peligrosa

El alimento retorna a la cocina en menos de 2 horas

$12 \quad 100$

Clave 5. Usar agua y materia prima seguros

Procedencia del agua es potable

Cuentan con instructivos para la recepción de materia prima

Lava las frutas y verduras siguiendo las indicaciones de inocuidad

Se visualiza indicaciones de lavado de frutas y hortalizas, con concentración de químicos.

En la rotación de alimentos se utiliza el sistema FIFO

Factores de riesgo para contaminación de alimentos según las 5 claves de la OMS

La distribución de servicios según el número de factores de riesgo para la contaminación de los alimentos por clave se presenta en la Tabla 4. De los 12 servicios visitados, seis (50\%) cumplieron adecuadamente los indicadores de la clave 1 ; tres servicios (25\%) cumplieron adecuadamente los indicadores de la clave 2 ; dos servicios $(16,66 \%)$ los indicadores de la clave 3; apenas un servicio (8,33\%) cumplió los indicadores de la clave 4 y dos servicios $(16,66 \%)$ cumplieron la totalidad de indicadores de la clave 5.

Tabla 4. Factores de riesgo para contaminación de alimentos según las 5 claves de la OMS. $\mathrm{n}=12$

\begin{tabular}{lllll}
\hline \multirow{2}{*}{ Claves } & Cero & Uno & No factores de riesgo & \\
& $6(50 \%)$ & $2(16,66 \%)$ & $2(16,66 \%)$ & $2(16,66 \%)$ \\
\hline Clave 1 & $3(25 \%)$ & $3(25 \%)$ & $5(41,67 \%)$ & $1(8,33 \%)$ \\
Clave 2 & $2(16,66 \%)$ & $10(83,34 \%)$ & - & - \\
Clave 3 & $1(8,33 \%)$ & $2(16,66 \%)$ & $5(41,67 \%)$ & $4(33,3 \%)$ \\
Clave 4 & $2(16,66 \%)$ & $3(25 \%)$ & $3(25 \%)$ & $4(33,3 \%)$ \\
Clave 5 & &
\end{tabular}

\section{Características socio-demográficas de los manipuladores}

Las características socio-demográficas de los manipuladores de alimentos se describen en la Tabla 5. Todos los manipuladores de alimentos era del sexo femenino entre 20 a 65 
años de edad (promedio \pm DE: $37,2 \pm 14,7$ años), estado civil soltera o casada con igual porcentaje $(50 \%)$, con escolaridad secundaria incompleta $(30,76 \%)$, marcado predominio del área urbana $(80,77 \%)$ y los años de servicio como manipulador de alimentos de un año o menos $(38,46 \%)$.

Tabla 5: Características sociodemográficas de los manipuladores. $n=26$

\begin{tabular}{|c|c|}
\hline Características & n (\%) \\
\hline \multicolumn{2}{|l|}{ Sexo } \\
\hline Femenino & $26(100)$ \\
\hline \multicolumn{2}{|l|}{ Edad (años) } \\
\hline $19-39$ & $13(52,0)$ \\
\hline $40-59$ & $9(36,0)$ \\
\hline$>60$ & $3(12,0)$ \\
\hline \multicolumn{2}{|l|}{ Estado civil } \\
\hline Soltero/a & $8(30,70)$ \\
\hline Casado/a & $8(30,70)$ \\
\hline Unión libre & $5(19,23)$ \\
\hline Viuda/Separada & $5(19,69)$ \\
\hline \multicolumn{2}{|l|}{ Número de hijos } \\
\hline Sin hijos & $7(26,92)$ \\
\hline 1 a 3 hijos & $15(57,69)$ \\
\hline Más de 4 hijos & $4(15,38)$ \\
\hline \multicolumn{2}{|l|}{ Procedencia (zona) } \\
\hline Urbana & $21(80,77)$ \\
\hline Rural & $5(19,23)$ \\
\hline \multicolumn{2}{|l|}{ Escolaridad } \\
\hline Primaria incompleta & $2(7,69)$ \\
\hline Primaria completa & $7(26,92)$ \\
\hline Secundaria incompleta & $8(30,76)$ \\
\hline Secundaria completa & $3(11,53)$ \\
\hline Universitaria incompleta & $2(7,69)$ \\
\hline Universitaria completa & $4(15,38)$ \\
\hline \multicolumn{2}{|l|}{ Promedio de ingreso mensual } \\
\hline <1 salario mínimo (SM) & $14(53,85)$ \\
\hline $1 \mathrm{SM}$ & $9(34,62)$ \\
\hline & $3(11,54)$ \\
\hline \multicolumn{2}{|c|}{ Antigüedad en el servicio como manipulador } \\
\hline$\leq 1$ año & $10(38,46)$ \\
\hline$>1$ y 3 años & $8(30,78)$ \\
\hline$\geq 4$ años & $8(30,78)$ \\
\hline
\end{tabular}

\section{DISCUSIÓN}

En este estudio se analizó la aplicación de las Buenas Prácticas de Manufactura (BPM) en la industria de alimentos en función a las "Cinco Claves de la OMS" para la inocuidad de los alimentos en todos los hospitales con servicios de alimentación del $\mathrm{V}$ departamento Caaguazú, Paraguay.

En relación a la clave 1 "Mantener la limpieza", el 66,7\% de los responsables de los servicios de alimentación reportó que los manipuladores de alimentos se lavaban las manos con la frecuencia recomendada. Sin embargo, el $66,67 \%$ de ellos no se lavaban las manos correctamente, y solo el $16,66 \%$ disponía de los insumos necesarios para ello. Estos hallazgos coinciden con un estudio en servicios de alimentación de escuelas, hospitales y casas de asistencia, en el que se encontró que el $60 \%$ del personal que servía los alimentos no se lavaba correctamente las $\operatorname{manos}^{(10)}$. Existen evidencias empíricas de la relación inversa entre el lavado de manos y la frecuencia de infecciones ${ }^{(11)}$, puesto que cualquier manipulación que se realice con las manos contaminadas, se convierte en un foco de infección y vehículo de transmisión de enfermedades ${ }^{(12)}$. En muchos casos en los que se realiza el lavado de manos, no se hace con una técnica correcta ni se consigue el resultado deseado(13). El lavado social es aquel que se realiza de forma convencional mediante la acción del agua y el jabón neutro, tiene efecto por arrastre y por la acción del jabón, no es eficaz en la eliminación de diversos microorganismos patógenos ${ }^{(14,15)}$. Como ha sido demostrado por varios autores, la bacteria adherida parece ser menos sensible a la limpieza 
y productos de desinfección que la bacteria en suspensión ${ }^{(16,17)}$. La simple tarea del lavado de las manos en los momentos adecuados y de la forma correcta es una medida que todo profesional sanitario debe realizar para prevenir una infección que podría causarle un daño grave al paciente incluso derivar hasta la muerte ${ }^{(18)}$. De los resultados obtenidos se deduce como una conclusión clara y precisa que hay que elevar el grado de educación sanitaria para fomentar el lavado de manos en el hospital y las estrategias deben contemplar programas de formación y educación sanitaria, facilitar los recursos materiales necesarios y contar con un buen apoyo institucional ${ }^{(13,19)}$.

Otro factor asociado al manipulador de alimentos es la higiene personal y la obligatoriedad de contar con uniforme de trabajo, se encontró que el $50 \%$ de los manipuladores tenían el uniforme de trabajo completo y los que portaban $20 \%$ estaban sucios. En un estudio en establecimientos dedicados a la preparación de platos preparados en el municipio de Madrid, encontraron que un $40 \%$ de los manipuladores trabajaban sin gorro $^{(20)}$. En las reglamentaciones vigentes, el uso del uniforme entre otros debe ser una práctica de rigor que cada operario debe cumplir, se debe tener una actitud de pulcritud y nitidez en las actividades que se lleven a cabo en los ambientes de trabajo.

Los procedimientos de limpieza son indispensables para disminuir los riesgos de contaminación, en el estudio se observó que el basurero de la cocina estaba sucio en el $41 \%$ de los servicios. Es extremadamente importante definir las fuentes de peligros durante la producción y distribución de alimentos para garantizar la higiene en los servicios de alimentación de los hospitales ${ }^{(21)}$. Los datos de la literatura indican que las malas prácticas de higiene en estos servicios pueden generar brotes de infecciones que pueden resultar en la muerte de los pacientes ${ }^{(22)}$. La implementación adecuada de buenas prácticas de manufactura (GMP/BPM), así como el sistema de análisis de peligros y puntos críticos de control (HACCP) juegan un papel clave en la prevención de las infecciones nosocomiales transmitidas por los alimentos ${ }^{(21)}$.

En relación a las instalaciones, el estudio mostró que el $84 \%$ de las aberturas que dan al exterior no ofrecen protección contra plagas, polvo u otros contaminantes constituyéndose en focos de contaminación que afectan negativamente la salud de las generaciones presentes y futuras. Los mayores incumplimientos en el local para cocina se encuentran en que no es apropiado para el uso que se destina, carecen de campanas extractoras y existen focos aislados de contaminación y suciedad ${ }^{(23)}$. Otro potencial factor de riesgo es la escasa iluminación en el $75 \%$ de los servicios y disposición limitada de sanitarios exclusivo para manipuladores $(41,66 \%)$, Para un buen trabajo de los operarios debe haber buena iluminación que ayuda a una mejor visualización de las líneas de procesamiento. Esta situación deja entrever el desconocimiento de la normativa existente sobre las condiciones básicas de higiene en la manipulación de alimentos. Las condiciones generales esenciales en los aspectos higiénico sanitarios y BPM en los servicios de alimentación establecidas en la Resolución 80/96 son disposiciones que deben ser aplicadas para asegurar la inocuidad de los alimentos y los organismos competentes son responsables de su implementación.

Los alimentos crudos y cocinados se mantenían separados en el $66,7 \%$, el $75 \%$ de los manipuladores utilizaba utensilios distintos para manipular alimentos crudos y listos para comer (clave 2). Los principios generales de higiene de los alimentos siguen procesos desde la producción primaria hasta el consumo final, resaltándose los controles en cada etapa. Todas las ETA son fundamentalmente evitables por medio de la modificación de la conducta de los manipuladores ${ }^{(24)}$. El 58,3\% aplicaba métodos apropiados en la degustación. El manipulador de alimentos es el principal agente de contaminación en el proceso de elaboración por las infracciones en el cumplimiento de las disposiciones legales ${ }^{(25)}$. Es necesario dar seguimiento a todos los esfuerzos para garantizar cambios permanentes y sostenibles en cada eslabón de la cadena alimentaria.

Todos los alimentos se cocinan hasta asegurar que la parte interna llegue a $70{ }^{\circ} \mathrm{C}$, pero solo $16,7 \%$ mantiene registros de la temperatura interna de los alimentos (clave 3 ). La mejor y única forma de asegurarse de que las bacterias han muerto y que los alimentos son seguros, es cocinarlos a la temperatura interna correcta, según las mediciones de un termómetro de alimentos. La clave es utilizar un termómetro para alimentos, según el Servicio de Seguridad e Inspección de los Alimentos (FSIS) del Departamento de Agricultura de los EE. UU. (USDA).

Con respecto a la clave 4 , el $16,7 \%$ de los alimentos con aliños como mayonesa, salsa de ajo se preparaba en pequeñas cantidades para limitar exposición a temperatura peligrosa, y 
el 58,3\% de los manipuladores realizaba la descongelación en el refrigerador. La exposición de los alimentos a temperaturas peligrosas conllevan contaminación microbiológica y deterioro de los alimentos (perdida de nutrientes y de características organolépticas), además de indicar que los encuestados no reconocen la importancia de la temperatura en el control de la supervivencia - crecimiento de los patógenos en los alimentos. La falta de conocimiento del personal de servicio de alimentos sobre las medidas de control de temperatura es particularmente preocupante en el servicio de alimentos porque el rango de temperatura peligrosa permite la supervivencia y el crecimiento exponencial de patógenos y toxinas transmitidas por los alimentos ${ }^{(26,27) \text {. }}$

En relación a la clave 5 , el $91,66 \%$ de los comedores hospitalarios poseen agua potable y el $25 \%$ de los manipuladores lava las frutas y verduras siguiendo las indicaciones de inocuidad. Una de las finalidades fundamentales del agua es el empleo en la higiene ${ }^{(19)}$. Es un hecho real que, por distintos medios, los alimentos se pueden contaminar y así convertir en transmisores de enfermedades, en detrimento de su función esencial como fuente de nutrimentos para una buena salud de quien los consume ${ }^{(28)}$. Las enfermedades diarreicas causadas por aguas y alimentos contaminados son una de las principales causas de morbilidad en todas las edades (FAO/WHO, 2005). Según los registros del sistema de información del Ministerio de Salud Pública y Bienestar Social, del 2007 al 2017 fueron reportados 138 brotes de ETA, enfermaron 2.345 personas y fallecieron $6\left({ }^{29}\right)$.

El $41,7 \%$ de los servicios cuentan con instructivos para la recepción de materia prima. En la rotación de alimentos, el $66,7 \%$ utilizaba el sistema FIFO. La primera oportunidad de asegurarse de que el alimento está en buenas condiciones es en el momento de la recepción de los productos. En ese momento es necesario asegurarse de que todos los productos vienen de fuentes autorizadas $y / 0$ de proveedores acreditados $y$ dignos de confianza. En un estudio realizado en 2006 y basado como el nuestro, en la observación directa y la realización de entrevistas junto con la aplicación de una "Guía para Evaluación de la Calidad" de uso interno, se encontró que sólo en un $18 \%$ de casos se comprobaba que los productos servidos se correspondían con los solicitados en la petición de compra.

En salud pública, para prevenir las consecuencias de la exposición a prácticas fraudulentas o inadecuadas en la industria alimentaria, es necesario asegurar la calidad y la inocuidad de los alimentos desde el origen y establecer cuáles son los estándares para su adquisición y posterior uso(30).

Otro aspecto que debe ser controlado en los servicios de alimentación es la formación sanitaria de los manipuladores. En los evaluados se encontró que un tercio de los manipuladores recibieron algún tipo de capacitación en educación sanitaria. Esta situación debe ser controlada en los servicios de alimentación porque permite prevenir en forma significativa los problemas sanitarios originados por desconocimiento de las condiciones seguras para el manejo adecuado de los alimentos en el proceso productivo ${ }^{(31)}$. En dos hospitales de Alejandría (Egipto) ${ }^{(32)}$, se evaluó el conocimiento de las prácticas de seguridad y alimentación de 23 personas que manipulaban alimentos antes y después de un programa de capacitación sobre inocuidad de los alimentos. Tras el entrenamiento hubo una mejoría significativa en todos los parámetros relacionados con el conocimiento y mejoras prácticas en materia de seguridad alimentaria. Los resultados de la mayoría de los estudios mostraron que la capacitación en higiene alimentaria de los manipuladores de alimentos mejoró los conocimientos sobre seguridad alimentaria( ${ }^{(33,34)}$. En consecuencia se puede afirmar que la escasa formación en protección de alimentos no puede garantizar la seguridad de los alimentos, tal como lo presenta Rodríguez con base a evaluaciones realizadas en servicios de alimentos en España ${ }^{(35)}$. Los esfuerzos para monitorear el comportamiento humano son complejos, y los factores motivacionales, perceptivos y ambientales deben ser tomados en consideración. Se debe monitorear la retención de conocimientos y los cambios de comportamiento entre los manipuladores de alimentos, de modo que se puedan diseñar e implementar cursos de actualización y capacitación dirigida ${ }^{(36)}$. La supervisión y control de rutina en los servicios de alimentación también deben ser efectuadas para garantizar que los procedimientos sean implementados de forma eficaz ${ }^{(37)}$. El fin de la alimentación hospitalaria ha de estar encaminado a proporcionar alimentos, que bajo un criterio higiénico sanitario, sean totalmente inocuos y seguros para los enfermos hospitalizados, ya que se trata de una población especialmente sensible frente a cualquier tipo de agresión ${ }^{(38)}$. 
La información generada en el presente trabajo tiene sus limitaciones, en nuestro caso el acceso a la cantidad total de manipuladores, no todos accedieron al estudio, además los servicios disponían de empleados que cumplían servicio en dos turnos durante los días hábiles y otro grupo (las franqueras) trabajaban solo los fines de semana y feriados, dificultaba el seguimiento; y las respuestas compiladas de los manipuladores fueron informados por los mismos, constituyéndose en prácticas reportadas, lo que también puede introducir algún sesgo en los comportamientos de los manipuladores estudiados.

La observación real de la preparación de alimentos habría sido beneficiosa, a fin de confirmar si el conocimiento y las actitudes expresadas en las respuestas fueron en realidad puestos en práctica. Además, las prácticas positivas podrían haber sido confirmadas por investigaciones microbianas.

Se sugiere como medida de control la capacitación permanente del manipulador de alimentos sobre BPM para la toma de conciencia de los riesgos, las reformas organizativoestructurales y la supervisión periódica por parte de las autoridades competentes de los entes involucrados, a fin de garantizar la inocuidad de los alimentos, requisito inherente a la salud de los consumidores.

Financiamiento: "Este Proyecto con código INV15-558 fue financiado por el CONACYT través del Programa PROCIENCIA con recursos del Fondo para la Excelencia de la Educación e Investigación - FEEI".

\section{REFERENCIAS BIBLIOGRAFICAS}

1. Kopper G. Enfermedades transmitidas por alimentos y su impacto socioeconómico. Estudio de caso en Costa Rica, El Salvador, Guatemala, Honduras y Nicaragua [Internet]. Costa Rica: FAO; 2009. [citado 22 Julio 2010]. Disponible en: http://www.fao.org/3/a-i0480s.pdf

2. Sánchez $R$, Gómez $F$. Evaluación de la organización y funcionamiento del servicio de alimentación en un hospital universitario. [Tesis]. Asunción: Universidad Católica de Asunción; 2016.

3. Moreno M, Alarcón A. Higiene alimentaria para la prevención de trastornos digestivos infecciosos y por toxinas. Rev. Med. Clin. Condes [internet]. 2010 (citado 22 Julio 2010); 21(5):749-55. Disponible en: www.clinicalascondes.com/areaacademica/pdf/MED.../749_755_Dr_Moren o.pdf/

4. Fernández N. Manual de investigación de brotes de enfermedades de origen alimentario. Asunción: Programa de Protección de Alimentos en la Ciudad de Asunción; 1998.

5. Organización Mundial de la Salud. Inocuidad de los alimentos, temas de salud [internet]. Ginebra: OMS; 2017. (citado 22 Julio 2010) Disponible en: http://www.who.int/mediacentre/factsheet s/fs399/es/

6. Organización Mundial de la Salud. Manual sobre las cinco claves para la inocuidad de los alimentos. [internet]. Ginebra: OMS; 2007 (citado 07 abril 2015). Disponible en: http://www.who.int/foodsafety/publication s/consumer/manual_keys_es.pdf

7. Mercosur/GMC/Res No 80/96. Reglamento técnico del Mercosur sobre las condiciones higiénico-sanitarias y de buenas prácticas de fabricación para establecimientos elaboradores/industrializadores

de alimentos [Internet]. Brasilia: GMC; 1996. [citado 15 Marzo 2017]. Disponible en: http://www.puntofocal.gov.ar/doc/r_gmc_ 80-96.pdf

8. Ramos $\mathrm{P}$, Araya S, Aranda $\mathrm{C}$ y Aquino $\mathrm{S}$. Condiciones sanitarias y factores de riesgo de contaminación alimentaria en comedores de Hospitales Públicos de Asunción. Asunción: Universidad Nacional de Asunción; 1998.

9. U.S. Department of Agriculture, Food and Nutrition Service, \& National Food Service Management Institute. The University of Mississipi. HACCP-based standard operating procedures (SOPs). [internet]. Mississipi University, MS: Author; 2005 Jun.115p. Disponible en: https://www.nj.gov/dcf/policy_manuals/O OE-I-A-1-48_A-1.doc

10. Martínez Tome $M$, Vera AM, Murcia MA. Improving the control of food production in catering establishment, with particular reference to the safety of salads. Food control [internet] 2000 (citado Diciembre 2000); 11(6):437-45. Disponible en: https://www.sciencedirect.com/science/.../ S0956713500000062

11. Tenias JM, Mayordomo ML, Benavent M, San Félix MA, García RA. Impacto de una intervención educativa para promover el lavado de manos y el uso racional de guantes en un hospital comarcal. Revista de calidad asistencial [internet]. 2009 (citado 1 de febrero 2009); 24(1):36-41. Disponible en: www.academia.edu/.../Impacto_de_una_in tervención_educativa_para_promover_el_l.

12. Barragen Gill P, Martín F, Martínez Espin MD, Rodríguez Alfaro $M$. Técnicas de enfermería y lavado de manos. Enfermería 
Integral. [internet]. 1998 (citado 4 mayo 2011); 9: 38-42. Disponible en: https://es.slideshare.net/jennie_mayaute/l avado-de-manos-7840586

13. Elola Vicente P. Higiene de las manos en el personal sanitario. Como aumentar y evaluar su cumplimiento. Todo Hospital. 2005; 214: 80-6.

14. Bayo Izquierdo MC. La higiene de manos y su importancia en la prevención de infecciones nosocomiales. Ciber Revista [Internet] Julio-Agosto 2013. [citado 10 ene 2016]; 32(4). Disponible en: http://www.enfermeriadeurgencias.co $\mathrm{m} /$ ciber/julio2013/pagina7.html

15. Sala A, Martínez Hernández J. Los trabajadores sanitarios necesitan más formación sobre la higiene de manos. Revista de Calidad Asistencial [Internet]. 2014 Nov-Dic (citado 10 ene 2016); 29(6):322-3. Disponible en: http://www.elsevierinstituciones.com/fiche ros/pdf/256/256v28n05a90231045pdf001. pdf

16. Frank JF and Koffi RA. Surface-Adherent Growth of listera monocytogenes is associated with increased resistance to surfactant sanitizers and heat. Journal of Food Prot. [internet].1990 (citado 1 de febrero 2009). 53: 550 - 54. Disponible en:

http://www.scirp.org/(S(351jmbntvnsjt1aa dkposzje))/reference/ReferencesPapers.asp X?ReferenceID $=1575710$

17. Stopfort JD, Yoon Y, Barmpalia JM, Samelis J, Skandomis PN, Reduction of listera monocytogenes and Eschercchia coli 0157:H7 in beef carcass wash water and on model equipment surfaces. Food Microbiol. 2003; 20: $651-60$.

18. Organización Mundial de la Salud. Guía de la OMS sobre Higiene de Manos en la Atención de la Salud: Resumen. Primer Desafío Global de Seguridad del Paciente. Una Atención Limpia es una Atención Segura. Génova, 2009: 80.

19. Jiménez Sesma ML, Pardo Vicastilo V. El impacto actual del lavado de manos. Medicina naturista. [internet].2008; 2(2): 77-83. Disponible

en: https://dialnet.unirioja.es/descarga/articul o/2574582.pdf

20. Sánchez C, Soldevilla A, Martín R y Sanz B. Estudio de las condiciones higiénico sanitarias de los establecimientos dedicados a la preparación de platos preparados en el municipio de Madrid. Aliment [internet] 1992; 229 (Enero Febrero):55 -60. Disponible en: https://dialnet.unirioja.es/servlet/autor?co digo $=1449587$

21. Konecka-Matyjek E, Krygier B, Tomczuk K, Stos $\mathrm{K}$, Jarosz M. National monitoring study on microbial contamination of foodcontact surfaces in hospital kitchens in Poland. Ann Agric Environ Med.[internet]
2012 (citado mayo 2012); 19(3):457-463. Disponible

en: https://www.ncbi.nlm.nih.gov/pubmed/23 020039

22. U.S. Food and Drug Administration, Center for Food Safety and Applied Nutrition. FDA report on the occurrence of foodborne illness risk factors in selected institutional foodservice, restaurant, and retail food store facility types. College Park, MD: FDA; 2004.

23. Arias $C$, Blanco N, Rodríguez A, Tardón A y Cueto $A$. Condiciones higiénico - sanitarias de comedores escolares del municipio de Oviedo. Rev. Esp. Salud Publica [internet].1998 (citado 19 julio 2018); 72(6): 571-81. Disponible en: http://scielo.isciii.es/pdf/resp/v72n6/come dores.pdf

24. Greig JD, Todd EC, Barttleson CA, Michaels BS.. Outbreaks where food workers have been implicated in the spread of foodborne disease. Part 1. Description of the problem, methods, and agents involved. J Food Prot 2007; 70(7): 1752-61.

25. Vázquez de Plata GE, Gómez de Avellaneda E, Gamboa Delgado EM. Condiciones higiénico - sanitarias de los servicios de alimentación en instituciones infantiles del Instituto Colombiano de Bienestar Familiar de Bucaramanga, Colombia. RCAN Rev Cubana Aliment Nutr [internet] 2007 (citado 13 enero 2015); 17(1):23-33. Disponible en: www.revicubalimentanut.sld.cu Contenidos $>$ Vol 17 No.1/RCAN.

26. Todd EC, Greig JD, Bartleson CA, Michaels BS, Outbreaks where food workers have been implicated in the spread of foodborne disease. Part 4. Infective doses and pathogen carriage. Journal of Food Protection [internet].2008. (citado Noviembre 2008); 71(11): 2339 - 73. Disponible en: jfoodprotection.org/doi/abs/10.4315/0362$028 X-70.7 .1752$

27. Webb $M$ and Morancie A. Food safety knowledge of foodservice workers at a university campus by education level, experience, and food safety training. Food control [internet]. 2015 (citado 9 septiembre 2014); 50: 259-64. Disponible en:

http://www.stat.wmich.edu/naranjo/article s/kendall2.pdf

28. Pérez-Silva García MC, Belmonte Cortes S, Martínez Corral J. Estudio microbiológico de los alimentos elaborados en comedores colectivos de alto riesgo. Rev Esp Salud Pública 1998 (citado: 24 julio 2018); 72(1): 67-75. Disponible en: http://scielo.isciii.es/scielo.php?script=sci arttext\&pid=S1135-57271998000100008

29. Ministerio de Salud Pública y Bienestar Social, Dirección General de Vigilancia de la Salud, Unidad de gestión de datos. 
Datos del año 2007 al 2017. Disponible en: http://vigisalud.mspbs.gov.py

30. Andrade W, Gómez R, Reyna O, Saldaña R. Informe de Evaluación del Departamento de Nutrición Hospital Iquitos Cesar Garayar García. [Internet]. Perú: Septiembre 2006 [citado 27 ene 2009]. Disponible en: http://www.cmploreto.com/hi/main/descar gas/calidad/4.pdf

31. Lazarević K, Stojonovic D, Bagdonovic DC, Dolicanin ZC. Hygiene Training of food handlers in hospital setting: Important factor in the prevention of nosocomial infections. Cent Eur J Public Health [internet].2013 (citado: set 2013); 21 (3): 146-9. Disponible en: https://www.ncbi.nlm.nih.gov/pubmed/24 344539

32. Buccheri C, Casuccio A, Giammanco S, Giammanco M, La Guardia M, Mammina C. Food safety in hospital: knowledge, attitudes and practices of nursing staff of two hospitals in Sicily, Italy. BMC Health Services Research [internet]. 2007 (citado: 3 abril 2007); 3(7):45-56. Disponible en: https://www.ncbi.nlm.nih.gov/pubmed/17 407582

33. Askarian M, Kabir G, Aminbaig M, Memish ZA, Jafari P. Knowledge, attitudes, and practices of food service staff regarding food hygiene in Shiraz, Iran. Infect Control Hosp Epidemiol. [internet]. 2004 (citado: enero 2004); 25(1):16-20. Disponible en: https://www.ncbi.nlm.nih.gov/pubmed/14 756213
34. Donkor E, Kayang J, Quaye J, Akyeh ML. Application of the WHO keys of safer food to improve food handling practices of food vendors in a poor resource community in Ghana. Int J Environ. Res Public Health [internet] 2009 (citado: Nov 2009); 6(11):2833-2842. Disponible en: www.mdpi.com/journal/ijerph y en https://www.ncbi.nlm.nih.gov/pmc/articles /PMC2800067/

35. Rodríguez JJ. Los costes ocultos de la calidad y la seguridad en los comedores escolares. [internet]. (citado: 10 set 2003). Disponible en: http://www.consumaseguridad.com/web/e s/sociedad_y/consumo/2002

36. Soon JM, Baines R, Seaman P. MetaAnalysis of Food Safety Training on Hand Hygiene Knowledge and Attitudes among Food Handlers. Journal of Food Protection, [internet]. 2012 (citado: abril 2012); 75(4): 793-804. Disponible en: https://www.ncbi.nlm.nih.gov/pubmed/22 488073

37. Munhoz PM, Pinto JPdAN, Biodi GF. Conhecimento sobre boas práticas por parte dos manipuladores. Hig Aliment 2008; 22(166/167):29-31.

38. Ruiz Santaolalla AT. Nutrición hospitalaria. Anales de la Real Academia de Ciencias Veterinarias de Andalucía Oriental. 1999; 12: 35-48. Disponible en: http://www.insacan.org/racvao/racvaopubl icacionesanales.html 